\title{
Motion Cueing for Stall Recovery Training in Commercial Transport Simulators
}

AIAA-2019-0979

Peter M. T. Zaal ${ }^{1}$

January 8, 2019

${ }^{1}$ San José State University

NASA Ames Research Center 


\section{Table of Contents}

1. Introduction

2. Methodology

3. Experiments

4. Conclusions

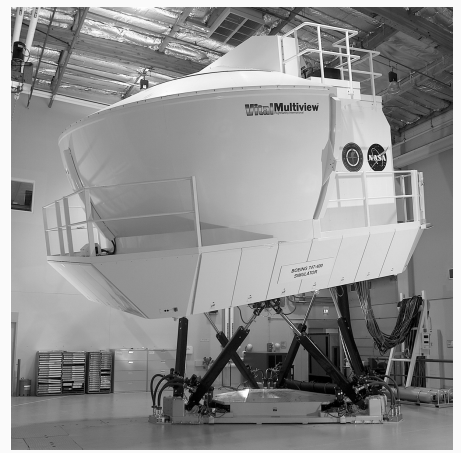




\section{Introduction}

1. Pilots are required to perform full stall recovery training in simulators starting this year

2. Historically, training simulators were not equipped for this

3. Post-stall aircraft models and representative motion cues need to be implemented

\section{Research Goal}

Develop motion cueing strategies for stall recovery training in commercial training simulators 


\section{Simulators}

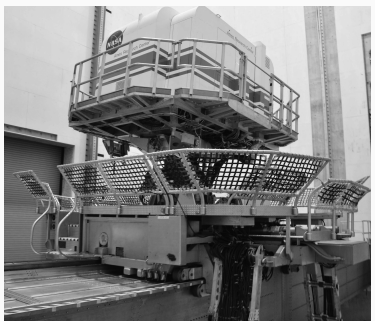

1. Largest vertical motion simulator

2. Transport Cab with sidestick

3. Electric + hydraulic motion system

4. Mid-size transport aircraft dynamics

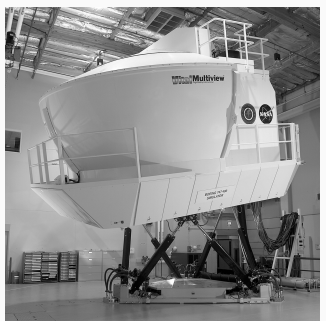

1. Equivalent to level-D certified

2. B747-400 cockpit with control column

3. Hydraulic hexapod motion system

4. Very-large transport aircraft dynamics 


\section{Transfer of Training}

Transfer-of-training paradigm most valid to study the training effectiveness of motion

Limitations of past research:

1. Most previous experiments performed with only slightly better motion in transfer condition (quasi transfer)

2. No good understanding and bad reporting of motion settings

3. Mostly outcome-based performance variables for measures 


\section{Pilot Modeling}

Pilot model parameters give insight into the use of visual and motion cues

Limitations of past research:

1. Single-axis

2. Time-invariant

3. Very structured tracking tasks

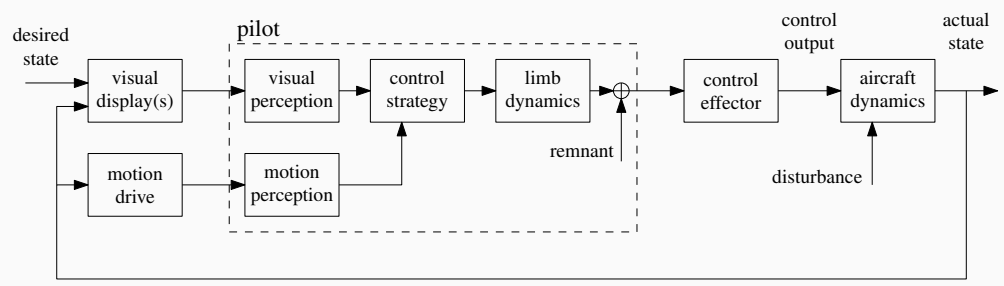




\section{Motion Cueing Strategy}

1. Simulators have limited motion space

2. Accelerations at pilot station need to be attenuated

3. Center of gravity linear accelerations require most motion space

\section{Approach}

Eliminating the center-of-gravity linear accelerations allows for a significant increase of the fidelity of remaining motion cues

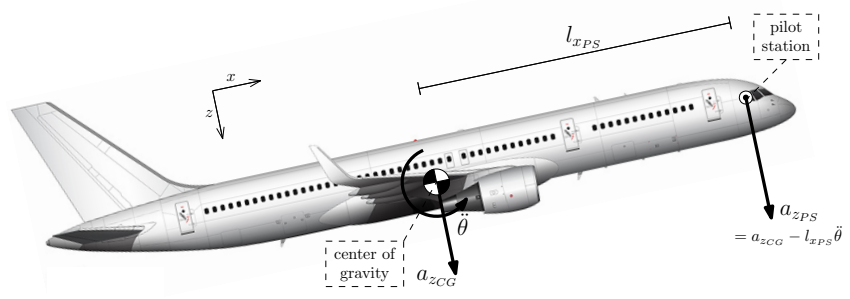




\section{Motion Cueing Strategy}

Limitations:

1. No sustained g-loads

2. No deceleration cue

3. No turn coordination 


\section{Experiments}

1. Effects of False Tilt Cues on the Training of Manual Roll Control Skills (AIAA-2015-0655)

2. Effects of Heave Motion Components on Pitch Control Behavior (AIAA-2016-3371)

3. Effects of Motion Cues on the Training of Multi-Axis Manual Control Skills (AIAA-2017-3473)

4. Time-Varying Manual Control Identification in a Stall Recovery Task under Different Simulator Motion Conditions (AIAA-2018-2936)

5. Adaptive Hexapod Simulator Motion based on Aircraft Stability (AIAA-2019-xxxx)

6. Verification of a Motion Cueing Strategy for Stall Recovery Training in a Commercial Transport Simulator (AIAA-2019-0426) 


\section{Experiment 1 - AIAA-2015-0655}

Goal:

Investigate the effects of false tilt cues on training and transfer of training of manual roll control skills

\section{Approach:}

1. Vertical Motion Simulator

2. Transfer-of-training experiment

3. Roll tracking task and multi-modal pilot modeling

4. Three training conditions: no motion, roll motion only, reduced coordinated roll motion

5. Transfer to full coordinated motion

6. Nineteen general aviation pilots

\section{Results:}

Pilots training with false tilt cues had significantly higher performance during training and after transfer 


\section{Experiment 2 - AIAA-2016-3371}

\section{Goal:}

Investigate if a different weighting of pilot station heave motion components allowed for control behavior closer to that in real flight

\section{Approach:}

1. Vertical Motion Simulator

2. Pitch tracking task and multi-modal pilot modeling

3. Nine different motion conditions

4. 21 general aviation pilots

\section{Results:}

Pilot control behavior in conditions with higher levels of rotational pitch heave was closer that in real flight 


\section{Experiment 3 - AIAA-2017-3473}

Goal:

Investigate the effects of two different hexapod motion configurations on the training and transfer of training of a multi-axis control task

\section{Approach:}

1. Vertical Motion Simulator

2. Transfer-of-training experiment

3. Roll-pitch tracking task and multi-modal pilot modeling

4. Two training conditions: baseline and enhanced hexapod

5. Transfer to full motion

6. Twenty general aviation pilots

\section{Results:}

Enhanced motion allowed pilots to generate less visual lead, control with higher gains, and have better disturbance-rejection performance at the end of training 


\section{Experiment 4 - AIAA-2018-2936}

Goal:

Investigate the effects of different motion cueing strategies on pilot control behavior in a stall recovery task

\section{Approach:}

1. Vertical Motion Simulator

2. Stall tracking task and single-loop time-varying pilot modeling

3. Four motion conditions: no motion, generic hexapod, enhanced hexapod, and full motion

4. Seventeen general aviation pilots

\section{Results:}

Pilot performance was highest for the enhanced and full motion conditions. Pilot control behavior under enhanced hexapod motion was most similar to that under full motion 


\section{Experiment 5 - AIAA-2019-xxxx}

Goal:

Development of an adaptive motion algorithm based on aircraft stability

\section{Approach:}

1. Vertical Motion Simulator

2. Two consecutive tasks: heading capture and stall recovery task

3. Pilot performance

4. Four motion conditions: generic, enhanced, adaptive, and full motion

5. Nineteen general aviation pilots

\section{Results:}

Normalized motion ratings reveal the adaptive motion to be most similar to the generic motion for the heading capture task, and most similar to the enhanced motion in the stall recovery tasks 


\section{Experiment 6 - AIAA-2019-0426}

Goal:

Verify the enhanced motion cueing strategy for improved stall recovery training on a commercial training simulator

\section{Approach:}

1. B747-400 level-D-certified full flight simulator

2. High-altitude stall recovery task

3. Three motion conditions: no motion, baseline motion, enhanced motion

4. Two aircraft dynamic conditions: baseline and enhanced

5. Eight airline pilots 


\section{Experiment 6 Results}

Maximum roll:

1. Significantly higher with enhanced dynamics

2. Significantly lower with higher fidelity motion

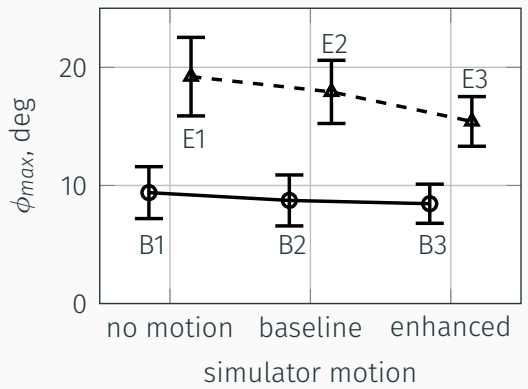

Altitude loss:

1. No significant differences

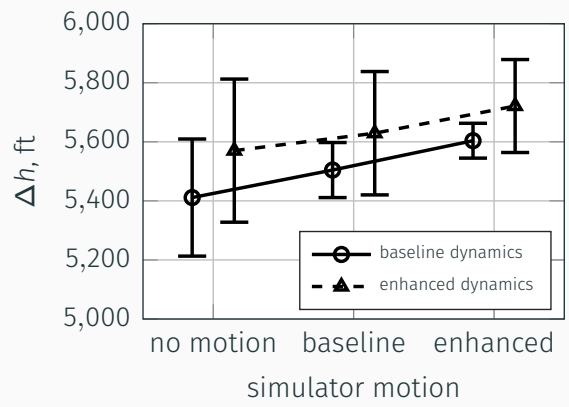




\section{Experiment 6 Results}

Minimum load factor:

1. Significantly higher with enhanced motion

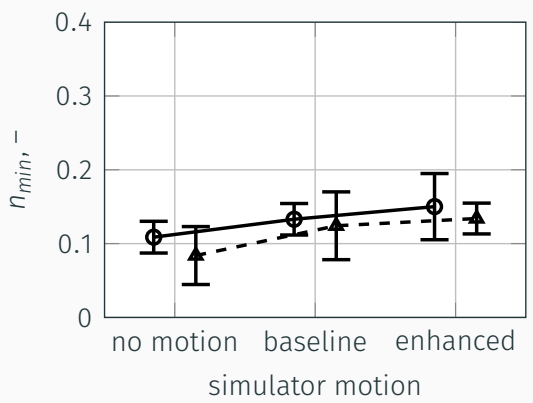

Maximum load factor:

1. No significant differences

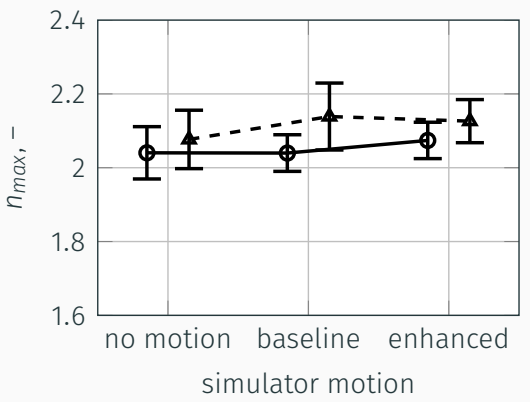




\section{Experiment 6 Results}

Additional stick shakers:

1. Significantly lower with enhanced motion

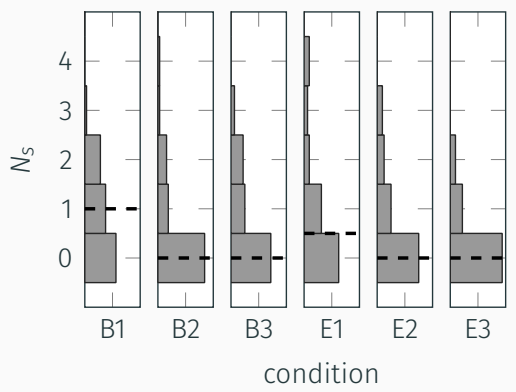

Maximum airspeed:

1. Significantly higher with enhanced motion

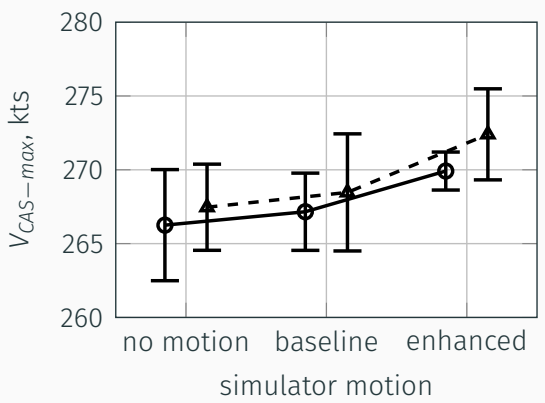




\section{Conclusions}

1. Better stall recovery performance with enhanced motion

1.1 Lower maximum roll

1.2 Less additional stick shakers

1.3 Higher minimum load factor

1.4 Higher maximum airspeed

2. Relatively minor enhancements allow for better stall recovery performance and potentially better training

3. Significant advancements were made with applying the transfer of training paradigm and time-varying pilot modeling techniques 
Questions?

peter.m.t.zaal@nasa.gov 